Proceeding Series of the Brazilian Society of Computational and Applied Mathematics

\title{
Projeto de Controlador Robusto via Programação Linear
}

\section{Leiliane Borges Cunha ${ }^{1}$}

Universidade Federal do Pará-UFPA, Programa de Pós-Graduação de Engenharia ElétricaPPGEE-UFPA.Rua Augusto Corrêa, 01, Guamá 66075-110, Belém - Pará.

\section{Anderson de França Silva ${ }^{2}$}

Universidade Federal do Pará-UFPA, Programa de Pós-Graduação de Engenharia ElétricaPPGEE-UFPA. Rua Augusto Corrêa, 01, Guamá 66075-110, Belém - Pará.

\section{José Augusto Lima Barreiros}

Universidade Federal do Pará-UFPA, Programa de Pós-Graduação de Engenharia ElétricaPPGEE-UFPA. Rua Augusto Corrêa, 01, Guamá 66075-110, Belém - Pará.

\section{Antônio da Silva Silveira ${ }^{4}$}

Universidade Federal do Pará-UFPA, Programa de Pós-Graduação de Engenharia ElétricaPPGEE-UFPA. Rua Augusto Corrêa, 01, Guamá 66075-110, Belém - Pará.

\section{Walter Barra Junior}

Universidade Federal do Pará-UFPA, Programa de Pós-Graduação de Engenharia ElétricaPPGEE-UFPA. Rua Augusto Corrêa, 01, Guamá 66075-110, Belém - Pará.

Resumo.Este trabalhoapresenta o projeto de um controlador robusto via programação linear em comparação com uma técnica de projeto convencional via posicionamento de polos. Os controladores foram aplicados à regulação de um sistema linear invariante no tempo. Testes foramrealizados a fim de verificar o desempenho dos controladores projetados.

Palavras-chave. Programação linear, motor CC, estabilidade, desempenho, robustez.

\section{Introdução}

\footnotetext{
${ }^{1}$ leilianebc@hotmail.com,

2 silva.anso@gmail.com

3barreiro@ufpa.br

4asilveira@ufpa.br

${ }^{5}$ walbarra@ufpa.br
} 
Muitos projetos de controladores são realizados a partir de modelos obtidos linearizando-se um sistema em torno de um ponto de operação fixo. Sendo assim, diante de uma eventual variaçãode seusparâmetros nominais, o sistema pode apresentar uma dinâmica diferente da qual foi levada em consideração no momento do projeto do controlador e isto pode ocasionar a instabilidade do sistema. Neste âmbito, surge a necessidade de projetar controladores robustos que sejam capazes de manter a estabilidade dos sistemas diante das variações em seus parâmetros e manter a robustez dentro de uma faixa aceitável [1].

O problema de projetar controladores robustos para plantas com incertezas paramétricas vem sendo extensivamente estudado ao longo dos últimos anos focando principalmente a estabilidade e desempenho robustos [1]. Em [3], é proposta uma metodologia de projeto na qual leva-se em consideração as incertezas paramétricas do sistema representadas por intervalos reais. Os controladores são projetados via programação linear através de uma análise politópica das incertezas. Em [5], é apresentado um sistema linear invariante no tempo, sujeito às variações em seus parâmetros nominais representados por intervalos reais fechados.

Portanto, neste trabalho é apresentado o projeto de um controladorutilizando-se a metodologia proposta em [3] aplicada ao sistema descrito em [5]. Testes foram realizados a fim de validar a estratégia de controle proposta para regulação do sistema em estudo.

\section{Propósito}

Este trabalho destina-se a mostrar o desempenho robusto de um motor CC, sujeito a variações paramétricas, através do projeto de um controlador robusto usando técnicas de programação linear. Tais técnicas consideram as incertezas o sistema. Com a utilização desta técnica avançada de controle, espera-se reduzir o esforço de controle quando o sistema estiver operando fora de seu ponto de operação nominal.

\section{Métodos}

\subsection{Verificação da estabilidade robusta de um sistema com parâmetros incertos.}

Considere uma planta em malha aberta, que descreve a dinâmica de um sistema linear e invariante no tempo, cujos parâmetros são incertos, definida pela equação (1).

$$
G(s)=\frac{n(s)}{d(s)}=\frac{n_{n} s^{n}+n_{n-1} s^{n-1}+\cdots+n_{0}}{d_{n} s^{n}+d_{n-1} s^{n-1}+\cdots+d_{0}}
$$

Onde $n_{l}=\left[n_{l}{ }^{-}, n_{l}{ }^{+}\right]$e $d_{l}=\left[{d_{l}}^{-}, d_{l}{ }^{+}\right] \in R^{n}, l=0,1, \ldots, n$ são os coeficientes de valores incertos da planta, delimitados por um intervalo reais fechados.

Em relação à estabilidade robusta, um sistema é considerado robustamente estável quando, diante de pequenas variações em torno do seu valor nominal, o sistema consegue manter a estabilidade, respeitando os limites mínimos e máximos de variações em seus parâmetros [3]. 


\subsection{Projeto do controlador robusto via técnica de programação linear.}

Considerando a equação (1), de ordem $\mathrm{n}$ e um controlador $\mathrm{C}(\mathrm{s})$, de ordem $\mathrm{r}$, mostrado na equação (2).

$$
C(s)=\frac{n_{c}(s)}{d_{c}(s)}=\frac{a_{r} s^{n}+a_{r-1} s^{n-1}+\cdots+a_{0}}{b_{r} s^{n}+b_{r-1} s^{n-1}+\cdots+b_{0}}
$$

O polinômio característico em malha fechada é descrito pela equação (3),

$$
\begin{gathered}
G_{M F}(s)=\left(n_{n} a_{r}+d_{n} b_{r}\right) s^{n+r}+\left(n_{n} a_{r-1}+n_{n-1} a_{r}+d_{n} b_{r-1}\right. \\
\left.+d_{n-1} b_{r}\right) s^{n+r-1}+\cdots+\left(n_{0} a_{0}+d_{0} b_{0}\right)
\end{gathered}
$$

Sua resposta transitória deve seguir um padrão de desempenho transitório especificado no intervalo $\delta_{i}^{-} \leq \delta_{i} \leq \delta_{i}^{+}$, para todo i, conforme equação (4).

$$
\delta(s)=\delta_{n+r} s^{n+r}+\delta_{n+r-1} s^{n+r-1}+\cdots+\delta_{0}
$$

Assim, para um sistema apresentar estabilidade e desempenho robustos, a equação (3) deve apresentar o mesmo desempenho do polinômio da equação (4), como mostra o sistema de equação (5).

$$
\begin{gathered}
\delta_{n+r}=n_{n} a_{r}+d_{n} b_{r} \\
\delta_{n+r-1}=n_{n} a_{r-1}+n_{n-1} a_{r}+d_{n} b_{r-1}+d_{n-1} b_{r} \\
\cdot \\
\cdot \\
\cdot \\
\delta_{0}=n_{0} a_{0}+d_{0} b_{0}
\end{gathered}
$$

Logo, segundo [3], para o projeto do controlador robusto, formula-se um conjunto de inequações lineares, mostrado na equação (6), que restringe os coeficientes do controlador nos intervalos definidos. Com isso, o sistema em malha fechada possui seus polos dentro do espaço de raízes do polinômio intervalar desejado [4].

$$
\left[\begin{array}{c}
\delta_{n+r^{-}}^{-} \\
\delta_{n+r-1}^{-} \\
\vdots \\
\delta_{0}^{-}
\end{array}\right] \leq\left[\begin{array}{c}
n_{n} a_{r}+d_{n} b_{r} \\
n_{n} a_{r-1}+n_{n-1} a_{r}+d_{n} b_{r-1}+d_{n-1} b_{r} \\
\vdots \\
n_{0} a_{0}+d_{0} b_{0}
\end{array}\right] \leq\left[\begin{array}{c}
\delta_{n+r}^{+} \\
\delta_{n+r-1} \\
\vdots \\
\delta_{0}^{+}
\end{array}\right]
$$

A partir da equação (6), usa-se a técnica de programação linear definida em[3],conforme a equação (7).

$\min \mathrm{f}(\mathrm{x})$

Sujeito à

$$
\mathrm{Ax} \leq \mathrm{b}, \mathrm{A}=\left[\begin{array}{c}
\mathrm{A} \\
-\mathrm{A}
\end{array}\right], \mathrm{b}=\left[\begin{array}{c}
\mathrm{b}_{\max } \\
-\mathrm{b}_{\min }
\end{array}\right]
$$


Onde $\mathrm{f}(\mathrm{x})$ é uma função linear arbitraria em $\mathrm{x}$, $\mathrm{x}$ é o vetor com as variáveis do controlador a serem otimizadas, $b_{\min }$ e $b_{\max }$, correspondem as inequações correspondentes ao limites inferiores e superiores do sistema em malha fechada, respectivamente. A matriz A corresponde aos coeficientes do sistema a ser otimizado.

\subsection{Controle de posição de um motor CC usando um controlador Proporcional Derivativo (PD) Robusto.}

Com base na técnica descrita nos itens anteriores, projeta-se um controlador PD para o motor CC, cuja entrada é a tensão na armadura $V_{a}$, em volts, e a saída, a posição angular $(\theta)$, em radianos. Ressalta-se que a escolha do controlador PD ocorre porque a planta já apresenta um integrador natural. Assim, considere a matriz de estado intervalar (8) e o vetor de entrada intervalar (9) que representa o motor CC [5]. A Tabela 1 mostra os dados usados na simulação.

$$
\dot{\mathrm{x}}=[\mathrm{A}] \mathrm{x}(\mathrm{t})+[\mathrm{B}] \mathrm{u}
$$

Onde:

$$
\begin{gathered}
x(t)=\left[\begin{array}{l}
x_{1}(t) \\
x_{2}(t)
\end{array}\right]=\left[\begin{array}{l}
\theta(t) \\
\dot{\theta}(t)
\end{array}\right] \\
{[A]=\left[\begin{array}{cc}
0 & 1 \\
0 & \frac{-B_{e q}}{\left[I_{\text {min }}, I_{\text {max }}\right]}
\end{array}\right] e[B]=\left[\begin{array}{c}
0 \\
\frac{A_{m}}{\left[I_{\text {min }}, I_{\text {max }}\right]}
\end{array}\right]}
\end{gathered}
$$

Tabela 1: Parâmetros do Motor DC

\begin{tabular}{|c|c|}
\hline $\mathrm{I}=\left[\mathrm{I}_{\text {min }}, \mathrm{I}_{\text {max }}\right]$ & {$[7.764,8.327] \times 10^{-3} \mathrm{Kg} \cdot \mathrm{m}^{2}$} \\
\hline $\mathrm{B}_{\mathrm{eq}}$ & $0.08404 \mathrm{~N} \mathrm{~m} \mathrm{~s} / \mathrm{rad}$ \\
\hline $\mathrm{A}_{\mathrm{m}}$ & $0.1285 \mathrm{~N} \mathrm{~m} / \mathrm{V}$ \\
\hline
\end{tabular}

Na Tabela 1, I representa o momento de inércia intervalar do motor CC, $\mathrm{A}_{\mathrm{m}}$ é o ganho do atuador, $\mathrm{B}_{\mathrm{eq}}$ o coeficiente de atrito viscoso.Aplicando a transformada de Laplace, tem-se a função de transferência correspondente, dada pela equação (10).

$$
\frac{\theta}{V_{a}}=\frac{\beta_{0}}{s\left(s+\alpha_{1}\right)}
$$

Sendo: $\beta_{0}=\frac{A_{m}}{\left[I_{\min }, I_{\max }\right]}$ e $\alpha_{1}=\frac{B_{e q}}{\left[I_{\min }, I_{\max }\right]}$.

Assim,

$$
G_{M F}(s)=\frac{[15.43,16.55]}{s^{2}+[10.09,10.82] s}
$$

Agora, admite-se o controlador PD, descrito pela equação (12). 


$$
C(s)=\mathrm{K}_{d} S+K_{p}
$$

De acordo com a equação (3), o polinômio característico da planta em malha fechada, com seus polos associados, toma a forma mostrada na equação (13).

$$
G_{M F}(s)=\mathrm{s}^{2}+\left(\alpha_{1}+\beta_{0} \mathrm{~K}_{d}\right) s+\beta_{0} \mathrm{~K}_{p}
$$

Assim, para uma resposta transitória desejada, considerou-se um tempo de pico Tp, um sobressinal Mp, um fator de amortecimento $\zeta$ e a freqüência natural de oscilação Wn do sistema, conforme Tabela 2, cujos polos (autovalores) desejados associados com as especificações sãomostrados na Tabela 3.

Tabela 2: Especificações de desempenho para o motor CC

\begin{tabular}{|c|c|c|c|}
\hline $\mathrm{Tp}(\mathrm{s})$ & $\mathrm{Mp}(\%)$ & $\zeta$ & $\mathrm{Wn}(\mathrm{rad} / \mathrm{s})$ \\
\hline 0.40 & {$[3,6]$} & {$[0.6671,0.7448]$} & {$[10.5430,11.7701]$} \\
\hline
\end{tabular}

Tabela 3: Autovalores desejados para o Motor CC

\begin{tabular}{|l|l|}
\hline$\lambda_{\min }$ & $-8.7664 \pm 7.8540 \mathrm{i}$ \\
\hline$\lambda_{\max }$ & $-7.0335 \pm 7.8540 \mathrm{i}$ \\
\hline
\end{tabular}
(14).

Resultando em um polinômio característico desejado intervalar mostrado na equação

$$
\delta(s)=s^{2}+[8.41,10.81] s+[79.37,90.92]
$$

Agora, considerando-se a equação (5) e o sistema de inequações (6) com seus parâmetros intervalares, tem-se:

$$
\mathrm{b}_{\min }=\left[\begin{array}{c}
-1.6824 \\
-2.4143 \\
-1.6824 \\
-2.4143 \\
79.37 \\
79.37
\end{array}\right], \mathrm{A}=\left[\begin{array}{cc}
15.43 & 0 \\
15.43 & 0 \\
16.55 & 0 \\
16.55 & 0 \\
0 & 15.43 \\
0 & 16.55
\end{array}\right], \mathrm{x}=\left[\begin{array}{c}
\mathrm{K}_{\mathrm{d}} \\
\mathrm{K}_{\mathrm{p}}
\end{array}\right] \text { e } \mathrm{b}_{\max }=\left[\begin{array}{c}
0.7216 \\
-0.0102 \\
0.7216 \\
-0.0102 \\
90.92 \\
90.92
\end{array}\right]
$$

Usando a técnica de programação linear na condição da equação (7), obtemos o controlador robusto ótimo, mostrado na equação (15).

$$
G_{c}(s)=0.2576 s+7.203
$$

\section{Resultados}

Com o objetivo de analisar o desempenho dos controladores projetados, foi realizado um teste aplicando-se um sinal do tipo degrau na entrada do sistema. Neste teste, os controladores foram submetidos à três pontos de operação diferentes, isto é, a três valores para o momento de inércia. Conforme ilustrado na Figura 1, nota-se o bom desempenho de ambos os controladores. Para toda a faixa de pontos de operação, tanto o controlador robusto sintonizado via programação linear quanto o controlador obtido via técnica clássica foram capazes de manter as especificações de desempenho. Com o 
tempo de pico em torno de 0,35 segundos e máximo sobressinal em torno de $6 \%$.

Destaca-se que o esforço de controle aplicado pelo controlador robusto, no transitório, foi ligeiramente menor do que o controlador convencional, principalmente quando foi modificado o ponto de operação.
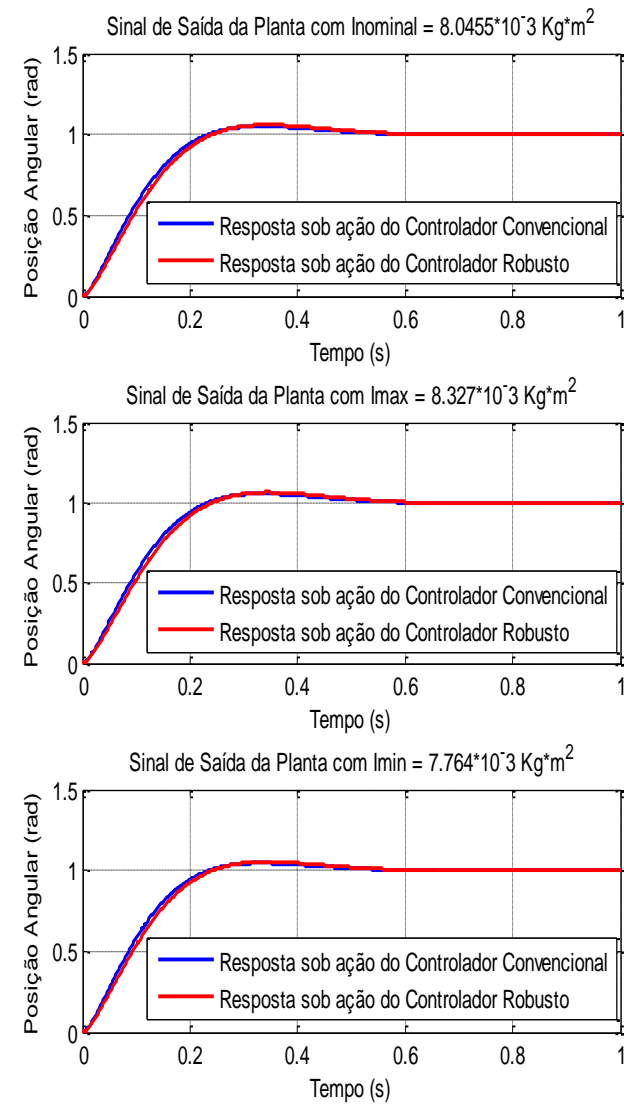
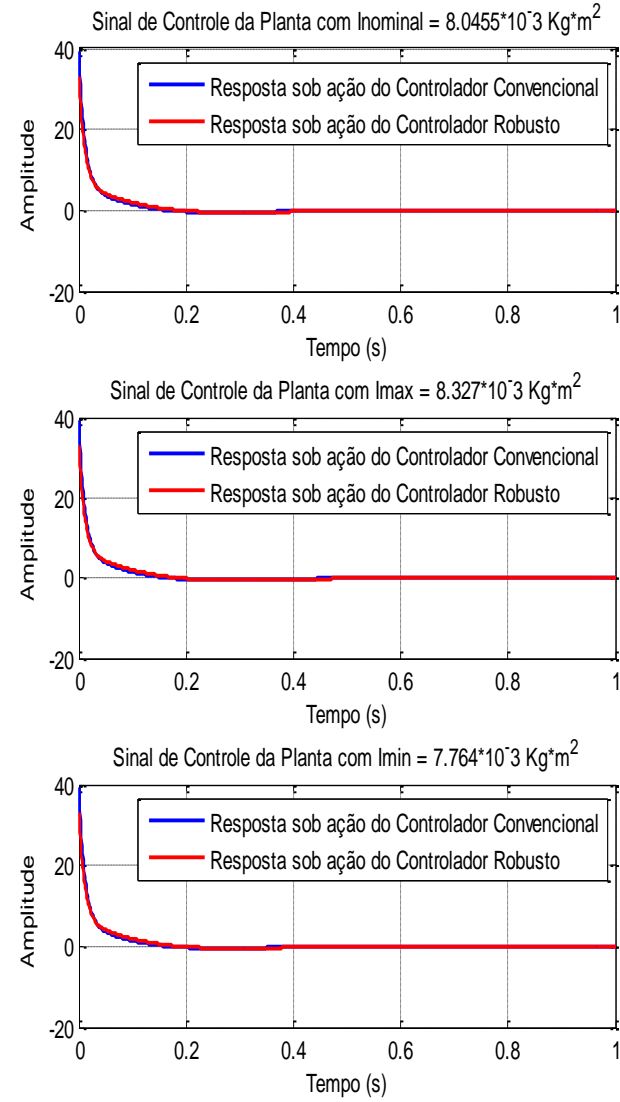

Figura 1: Sinal de Saída e do Esforço de Controle.

\section{Conclusão}

Os resultados apresentados permitem concluir,através da aplicabilidade da técnica de sintonização proposta neste artigo para o sistema em estudo,que o controlador robusto garantiu a robustez em desempenho e em estabilidade diante da variação paramétrica do sistema. Vale ressaltar a robustez da técnica clássica, a qual também apresentou um bom desempenho.

A aplicabilidade do método proposto para outros sistemas físicos ainda carece de maior investigação que inclua a comparação entre metodologias clássicas e avançadas com o objetivo de aproveitar melhor cada uma das técnicas, quando analisados os desempenhos dinâmicos diante das variações paramétricas dos sistemas físicos.

\section{Agradecimentos}

Os autores agradecem à Coordenação de Aperfeiçoamento de Pessoal de Nível Superior-CAPES pelo apoio financeiro dado a esta pesquisa e à Universidade Federal do 
Pará-UFPA por disponibilizar recursos humanos e materiais que propiciaram a realização deste trabalho.

\section{Referências}

[1] S. P. Bhattacharyya, H. Chapellat and L. H. Keel, Robust Control: The Parametric Approach, vol.1, (1995).

[2] C. A. Costa, Projeto e Avaliação de uma estratégia baseada em analise intervalar aplicada ao projeto de estabilizador de sistema de potencia robusto implementado e um sistema de geração de 10KVA, Dissertação de Mestrado, UFPA, (2013).

[3] L. H. Keel and S. P. Bhattacharyya, A linear programming Approach To Controller Design, IEEE Transactions on Automatic Control, California USA, (1997).

[4] A. D. S. Lordelo and P. A. V. Ferreira, Interval analysis and design of robust pole assignment controllers, In Proceedings of the 41th IEEE conference on decision and control, Las Vegas, (2002).

[5] A. D. S. Lordelo and H. A. Fazzolari, On interval goal programming switching surface robust design for Integral Sliding Mode Control, Control Engineering Practice, Cap.32,vol. 32,(2014). 